\title{
Switching control between three-coil and five-coil modes for six-pole active magnetic bearings
}

\author{
Satoshi UENO* and Changan JIANG* \\ * Department of Mechanical Engineering, College of Science and Engineering, Ritsumeikan University \\ 1-1-1 Nojihigashi, Kusatsu, Shiga, 525-8577, Japan \\ E-mail: sueno@se.ritsumei.ac.jp
}

Received: 9 June 2019; Revised: 29 July 2019; Accepted: 2 September 2019

\begin{abstract}
Six-pole active magnetic bearings (AMBs) have fewer poles than the more common eight-pole AMBs making it possible for them to be miniaturized and used in the development of small motors. In this paper, we propose a novel control method for the six-pole AMB, which switches between three-coil and five-coil modes. The three-coil mode excites three coils to generate a suspension force and reduce power consumption. In contrast, the five-coil mode excites five coils and can generate a larger maximum force than the three-coil mode. By switching between the two modes, it is possible to realize both an increase in the maximum suspension force and a reduction in power consumption. This paper derives the coil currents in the three- and five-coil modes, and introduces a switching method between them. The proposed method is verified with a test rig, and results show that the proposed method enables stable levitation with a lower current limit compared with the method that uses the three-coil mode alone. Moreover, a start-up procedure with a low current limit is proposed and experimentally verified.
\end{abstract}

Keywords : Active magnetic bearing, Six-pole stator, Control current, Power consumption, Switching control, Three-coil mode, Five-coil mode, Rotary machinery

\section{Introduction}

Improvements in durability and reductions in the noise of small motors have been requirements since a long time. Therefore, magnetically suspended motors have been developed (Asama et al., 2014; Kanebako and Okada, 2002; Osa et al., 2017). They have advantages such as no losses due to friction, no abrasion, and lubrication-free operation (Chiba et al., 2005). Furthermore, active magnetic bearings (AMBs) have the advantage of vibration suppression and highperformance control because they are capable of active control (Mizuno and Higuchi, 1990). The development of a smaller and relatively low cost AMBs has been proposed (Chen and Hsiao, 2017; Kakihara et al., 2005; Li et al., 2002; Yoshida et al., 2006). As a results, six-pole AMBs (Chiba et al., 2017; Ueno et al., 2004, 2007; Ueno, 2013) have been developed. More common eight-pole AMBs have more poles than six-pole AMBs, but are more difficult to miniaturize, thereby increasing costs. Moreover, the maximum force of six-pole AMBs is larger than that of three-pole AMBs with the same outer diameter of a rotor, because the magnetic flux density in the half of the rotor surface can be set to zero in six-pole AMBs. However, because the magnetic circuit of the six-pole AMBs is different in the $x$ - and $y$-directions; therefore, the calculation of coil currents becomes complex. To solve this problem, we have developed several control methods such as a minimum energy control method (Ueno et al., 2007) and a maximum bearing force control method (Ueno, 2013).

The minimum energy control method uses three coils located near the direction of the target bearing force. We refer to this method as the three-coil mode in this paper. This method can reduce copper losses; however, the maximum bearing force towards a pole face becomes $72 \%$ of the maximum bearing force towards the center of two poles. To solve this problem, the maximum bearing force control method has been developed. This method uses five coils, with the currents of the same magnitude applied to the three coils in the middle, while the coils on either side control the direction of the 
suspension force. With this method, nearly the same maximum bearing force can be obtained in all directions. However, this method uses more energy than the three-coil mode (Chiba et al., 2017).

To realize both low power consumption and large maximum force, we introduce a switching control method in this paper. When the bearing force is small, the coils are driven by the three-coil mode. If one of the coil currents reaches a limit value, the coil currents are switched to the five-coil mode. To enable smooth switching, a new calculation method in the five-coil mode was developed instead of the maximum bearing force control method. The proposed method was verified by numerical simulations and experiments. The results show that the proposed method allows for stable levitation with a lower current limit compared with other methods.

\section{Control method}

\subsection{Suspension force of six-pole AMB}

A coordinate system and the magnetic circuit of six-pole AMBs are shown in Fig. 1. Magnetic attractive forces at each pole are expressed as

$$
f_{k}=\frac{S}{2 \mu_{0}} B_{k}^{2} \quad k=1, \cdots, 6
$$

where $\mu_{0}$ is the permeability of air, $S$ is the cross section of the pole, and $k$ is the number of the poles. The sum of $B_{k}$ becomes zero. The $x$ - and $y$-components of the suspension force are calculated as follows

$$
\begin{aligned}
& f_{x}=f \cos \phi=\frac{\sqrt{3}}{2}\left(f_{6}+f_{5}-f_{2}-f_{3}\right)=\frac{\sqrt{3} S}{4 \mu_{0}}\left(B_{6}^{2}+B_{5}^{2}-B_{2}^{2}-B_{3}^{2}\right) \\
& f_{y}=f \sin \phi=f_{1}+\frac{1}{2} f_{2}-\frac{1}{2} f_{3}-f_{4}-\frac{1}{2} f_{5}+\frac{1}{2} f_{6}=\frac{S}{2 \mu_{0}}\left(B_{1}^{2}+\frac{1}{2} B_{2}^{2}-\frac{1}{2} B_{3}^{2}-B_{4}^{2}-\frac{1}{2} B_{5}^{2}+\frac{1}{2} B_{6}^{2}\right)
\end{aligned}
$$

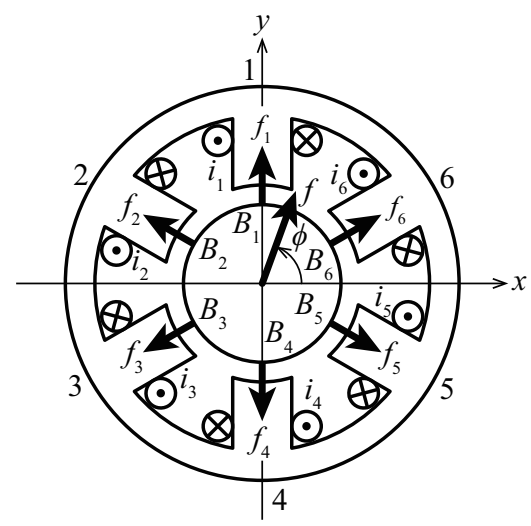

(a) Coordinate system of six-pole AMB.

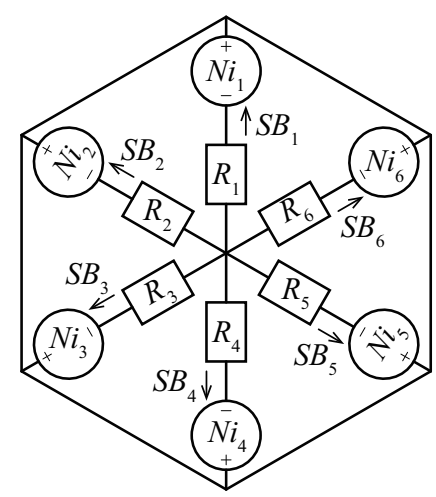

(b) Magnetic circuit of six-pole AMB.

Fig. 1 Coordinate system and magnetic circuit of six-pole AMB. Coils are driven by individual amplifiers, allowing the coil currents, $i_{k}$, to be controlled independently. $B_{k}$ and $f_{k}$ are the magnetic flux density and the attractive force between the stator and rotor at each pole, respectively. $f$ and $\phi$ denote the magnitude and angle of the suspension force, respectively. $N$ is the number of coil turns, and $R_{k}$ is the magnetic resistance of the air gap at each pole. The Magnetic resistance of the iron core and leakage flux are neglected in the magnetic circuit.

\subsection{Magnetic flux densities in three-coil mode}

First, we discuss the three-coil mode. In this mode, only three poles in the neighborhood of the target angle are excited for force generation. For example, if the target angle is $\pi / 3 \leq \phi<2 \pi / 3$, poles 1,2 , and 6 are excited, and $B_{3}, B_{4}$, and $B_{5}$ are set to zero. Therefore, the magnetic flux densities are derived by solving the following simultaneous equations:

$$
\begin{aligned}
& \hat{f} \cos \hat{\phi}=\frac{\sqrt{3} S}{4 \mu_{0}}\left(B_{6}^{2}-B_{2}^{2}\right) \\
& \hat{f} \sin \hat{\phi}=\frac{S}{2 \mu_{0}}\left(B_{1}^{2}+\frac{1}{2} B_{2}^{2}+\frac{1}{2} B_{6}^{2}\right)
\end{aligned}
$$




$$
B_{1}+B_{2}+B_{6}=0
$$

where $\hat{f}$ and $\hat{\phi}$ are the magnitude and angle of the target force, respectively. The solution is

$$
\begin{aligned}
& B_{k}=\alpha_{k} \sqrt{\frac{\mu_{0}}{S}} \sqrt{\hat{f}} \quad k=1,2 \\
& B_{6}=-B_{1}-B_{2}
\end{aligned}
$$

where

$$
\begin{aligned}
& \alpha_{1}=\sqrt{\frac{4}{5 \sqrt{3}}} \sqrt{\sqrt{3} \sin \hat{\phi}+\sqrt{3-8 \cos ^{2} \hat{\phi}}} \\
& \alpha_{2}=-\sqrt{\frac{4}{5 \sqrt{3}}} \sqrt{-\sqrt{13} \cos \left(\hat{\phi}+\tan ^{-1} \frac{3 \sqrt{3}}{5}\right)-\sqrt{3-8 \cos ^{2} \hat{\phi}}}
\end{aligned}
$$

When $B_{1}, B_{2}$, and $B_{6}$ are less than the maximum value, the target force can be generated. The other angles can be calculated by shifting coil currents as described later.

\subsection{Magnetic flux densities in five-coil mode with the same amplitude for three poles}

The target force can also be generated by using five poles. One of the sets of magnetic flux densities is obtained by setting three poles in the neighborhood of the target angle to the same magnitude. For example, if the target angle is $\pi / 3 \leq \hat{\phi}<2 \pi / 3, B_{2}$ and $B_{6}$ have the same magnitude as $B_{1}$. i.e., $B_{2}=B_{6}=-B_{1} . B_{3}$ and $B_{5}$ are adjusted according to the angle of the target force, and $B_{4}$ is set to zero. The simultaneous equations are expressed as

$$
\begin{aligned}
& \hat{f} \cos \hat{\phi}=\frac{\sqrt{3} S}{4 \mu_{0}}\left(B_{5}^{2}-B_{3}^{2}\right) \\
& \hat{f} \sin \hat{\phi}=\frac{S}{2 \mu_{0}}\left(2 B_{1}^{2}-\frac{1}{2} B_{5}^{2}-\frac{1}{2} B_{3}^{2}\right) \\
& B_{1}=B_{3}+B_{5}
\end{aligned}
$$

By solving the above equations, we find

$$
\begin{aligned}
& B_{k}=\beta_{k} \sqrt{\frac{\mu_{0}}{S}} \sqrt{\hat{f}} \quad k=1,3 \\
& B_{5}=B_{1}-B_{3}
\end{aligned}
$$

where

$$
\begin{aligned}
& \beta_{1}=\sqrt{\frac{4}{7}} \sqrt{\sin \hat{\phi}+\sqrt{1+\frac{4}{3} \cos ^{2} \hat{\phi}}} \\
& \beta_{3}=\frac{\beta_{1}}{2}-\frac{2 \cos \hat{\phi}}{\sqrt{3} \beta_{1}}
\end{aligned}
$$

In this method a larger suspension force can be generated. However, copper loss increases compared to the three-coil mode. Moreover, as the magnetic flux densities are different from the three-coil mode, they suddenly change when the mode is switched between the three-coil and five-coil modes. We introduce another method in the next section to avoid this problem. This method is referred to as the five-coil A mode. 


\subsection{Magnetic flux densities in five-coil mode with a saturated pole}

When the magnetic flux density of one pole reaches to its maximum in the three-coil mode, an additional two poles can be utilized to generate a larger force. However, when $\hat{\phi}=k \pi / 3$, it is impossible to generate additional suspension force because the next two poles are orthogonal to the target angle. Therefore, $\pi / 3<\hat{\phi}<2 \pi / 3$ is considered.

In this case, poles $1,2,3,5$, and 6 are excited, and pole 1 reaches to its maximum magnetic flux density denoted as $\bar{B}_{1}$. As $B_{1}$ is at its maximum, the attractive force at pole 1 becomes

$$
\bar{f}_{1}=\frac{S}{2 \mu_{0}} \bar{B}_{1}^{2}
$$

The AMB then has to generate the rest of the force by exciting poles $2,3,5$, and 6 , and the following relationships are derived.

$$
\begin{aligned}
& \hat{f} \cos \hat{\phi}=\frac{\sqrt{3}}{2}\left\{\left(f_{6}-f_{3}\right)+\left(f_{5}-f_{2}\right)\right\} \\
& \hat{f} \sin \hat{\phi}-\bar{f}_{1}=\frac{1}{2}\left\{\left(f_{6}-f_{3}\right)-\left(f_{5}-f_{2}\right)\right\}
\end{aligned}
$$

From Eqs. (19) and (20), we find

$$
\begin{aligned}
& f_{6}-f_{3}=\frac{1}{\sqrt{3}} \hat{f} \cos \hat{\phi}+\hat{f} \sin \hat{\phi}-\bar{f}_{1} \\
& f_{5}-f_{2}=\frac{1}{\sqrt{3}} \hat{f} \cos \hat{\phi}-\hat{f} \sin \hat{\phi}+\bar{f}_{1}
\end{aligned}
$$

In contrast, the forces generated by the couple of opposite poles are expressed as

$$
\begin{aligned}
& f_{6}-f_{3}=\frac{S}{2 \mu_{0}}\left(B_{6}^{2}-B_{3}^{2}\right)=\frac{2 S}{\mu_{0}}\left(\frac{B_{6}+B_{3}}{2}\right)\left(\frac{B_{6}-B_{3}}{2}\right)=\frac{2 S}{\mu_{0}} B_{b 63} B_{c 63} \\
& f_{5}-f_{2}=\frac{S}{2 \mu_{0}}\left(B_{5}^{2}-B_{2}^{2}\right)=\frac{2 S}{\mu_{0}}\left(\frac{B_{5}+B_{2}}{2}\right)\left(\frac{B_{5}-B_{2}}{2}\right)=\frac{2 S}{\mu_{0}} B_{b 52} B_{c 52}
\end{aligned}
$$

where we define $B_{b 63}$ and $B_{b 52}$ as bias flux and $B_{c 63}$ and $B_{c 52}$ as control flux, respectively. From Eqs. (21-24), $B_{c 63}$ and $B_{c 52}$ are derived as

$$
\begin{aligned}
& B_{c 63}=\frac{\mu_{0}}{2 S} \frac{1}{B_{b 63}}\left(\frac{1}{\sqrt{3}} \hat{f} \cos \hat{\phi}+\hat{f} \sin \hat{\phi}-\bar{f}_{1}\right) \\
& B_{c 52}=\frac{\mu_{0}}{2 S} \frac{1}{B_{b 52}}\left(\frac{1}{\sqrt{3}} \hat{f} \cos \hat{\phi}-\hat{f} \sin \hat{\phi}+\bar{f}_{1}\right)
\end{aligned}
$$

Next, $B_{b 63}$ and $B_{b 52}$ are discussed. As $B_{4}=0$, the magnetic flux densities satisfy the following relation.

$$
\bar{B}_{1}=-B_{2}-B_{3}-B_{5}-B_{6}=2\left(B_{b 63}+B_{b 52}\right)
$$

It then follows that

$$
B_{b 63}=-\frac{1}{2} \bar{B}_{1}-B_{b 52}
$$

When $B_{1}$ reaches to $\bar{B}_{1}$ in the three-coil mode, the magnetic flux density at pole 2 is calculated as

$$
B_{2}=\gamma \bar{B}_{1}
$$

where

$$
\gamma=\left\{\begin{array}{lc}
-\frac{1}{2}\left(1-\sqrt{3} \tan \hat{\phi}+\sqrt{3 \tan ^{2} \hat{\phi}-5}\right) & \frac{\pi}{3}<\hat{\phi}<\frac{\pi}{2} \\
-\frac{1}{2} & \hat{\phi}=\frac{\pi}{2} \\
-\frac{1}{2}\left(1-\sqrt{3} \tan \hat{\phi}-\sqrt{3 \tan ^{2} \hat{\phi}-5}\right) & \frac{\pi}{3}<\hat{\phi}<\frac{\pi}{2}
\end{array}\right.
$$


Moreover, $B_{5}$ is zero. Then, $B_{b 52}$ is obtained from Eqs. (24) and (29) as

$$
B_{b 52}=\frac{B_{5}+B_{2}}{2}=\frac{\gamma \bar{B}_{1}}{2}
$$

Finally, the magnetic flux densities are obtained as

$$
\begin{aligned}
& B_{2}=B_{b 52}-B_{c 52} \\
& B_{3}=B_{b 63}-B_{c 63} \\
& B_{5}=B_{b 52}+B_{c 52} \\
& B_{6}=B_{b 63}+B_{c 63}
\end{aligned}
$$

This method is called the 5-coil B mode.

\subsection{Coil currents}

A magnetic circuit of a six-pole AMB is shown in Fig. 1 (b). The magnetic resistance of air gaps is represented by

$$
R_{k}=\frac{1}{\mu_{0} S}\left\{g_{0}+x \sin \frac{(k-1) \pi}{3}-y \cos \frac{(k-1) \pi}{3}\right\} \quad k=1, \cdots, 6
$$

where $g_{0}$ is the air gap length when the rotor is located at the center. From the magnetic circuit, the following equation is derived.

$$
\left[\begin{array}{ccccc}
R_{1} & -R_{2} & 0 & 0 & 0 \\
0 & R_{2} & -R_{3} & 0 & 0 \\
0 & 0 & R_{3} & -R_{4} & 0 \\
0 & 0 & 0 & R_{4} & -R_{5} \\
R_{6} & R_{6} & R_{6} & R_{6} & R_{5}+R_{6}
\end{array}\right]\left[\begin{array}{c}
S B_{1} \\
S B_{2} \\
S B_{3} \\
S B_{4} \\
S B_{5}
\end{array}\right]=\left[\begin{array}{cccccc}
1 & -1 & 0 & 0 & 0 & 0 \\
0 & 1 & -1 & 0 & 0 & 0 \\
0 & 0 & 1 & -1 & 0 & 0 \\
0 & 0 & 0 & 1 & -1 & 0 \\
0 & 0 & 0 & 0 & 1 & -1
\end{array}\right]\left[\begin{array}{c}
N i_{1} \\
N i_{2} \\
N i_{3} \\
N i_{4} \\
N i_{5} \\
N i_{6}
\end{array}\right]
$$

Note the sum of the magnetic flux densities should be zero. Then, $B_{6}=-\left(B_{1}+B_{2}+B_{3}+B_{4}+B_{5}\right)$. The coil current can be obtained by multiplying pseudo-inverse of coefficient of currents.

$$
\left[\begin{array}{c}
i_{1} \\
i_{2} \\
i_{3} \\
i_{4} \\
i_{5} \\
i_{6}
\end{array}\right]=\frac{S}{N} \cdot \frac{1}{6}\left[\begin{array}{ccccc}
5 & 4 & 3 & 2 & 1 \\
-1 & 4 & 3 & 2 & 1 \\
-1 & -2 & 3 & 2 & 1 \\
-1 & -2 & -3 & 2 & 1 \\
-1 & -2 & -3 & -4 & 1 \\
-1 & -2 & -3 & -4 & -5
\end{array}\right]\left[\begin{array}{ccccc}
R_{1} & -R_{2} & 0 & 0 & 0 \\
0 & R_{2} & -R_{3} & 0 & 0 \\
0 & 0 & R_{3} & -R_{4} & 0 \\
0 & 0 & 0 & R_{4} & -R_{5} \\
R_{6} & R_{6} & R_{6} & R_{6} & R_{5}+R_{6}
\end{array}\right]\left[\begin{array}{c}
B_{1} \\
B_{2} \\
B_{3} \\
B_{4} \\
B_{5}
\end{array}\right]
$$

If the displacements are small and can be neglected, the coil currents are calculated by

$$
i_{k}=\frac{g_{0}}{\mu_{0} N} B_{k} \quad k=1, \cdots, 6
$$

\subsection{Implementation}

To reduce computation time, $\alpha_{k}, \beta_{k}$, and $\gamma$ are calculated beforehand and stored in lookup tables. The coil currents are obtained by the following procedure.

First, the target forces $\hat{f}_{x}$ and $\hat{f}_{y}$ are transformed to $\hat{f}$ and $\hat{\phi}$. Then, $\hat{\phi}$ is shifted from $\pi / 3$ to $2 \pi / 3$ by computing the remainder of $\hat{\phi}$ divided by $\pi / 3$, and adding $\pi / 3$ to the remainder. Then, the magnetic flux densities are computed from the lookup table of the three-coil mode and compared with the limit value, which is calculated from Eq. (39) and the current limit. If all the magnetic flux densities are less than the limit, they are adopted. If one of the magnetic flux densities is over the limit, they are computed again by using the five-coil A or B modes. Thus, temporary magnetic flux densities $\tilde{B}_{k}$ are determined. 
Next, the magnetic flux density at each pole is determined according to the range of $\hat{\phi}$ as

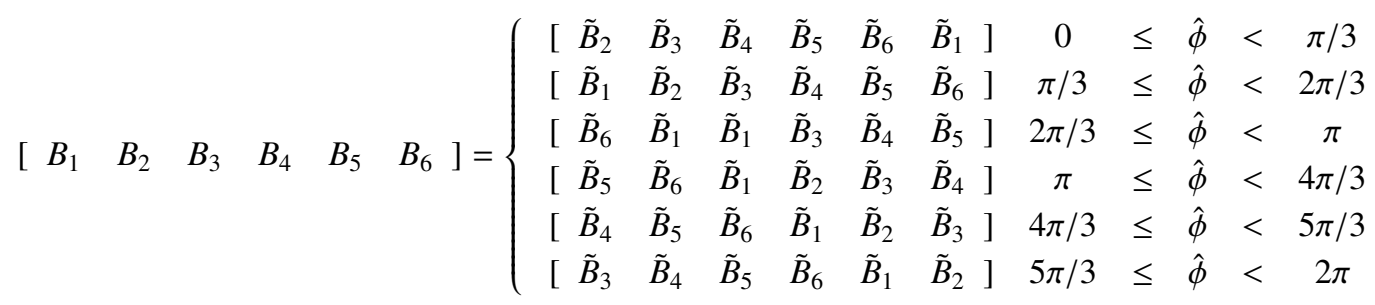

Then, the coil currents are calculated by Eq. (39).

\subsection{Numerical simulation}

The proposed methods were verified with numerical simulation. The parameters are listed in Table 1, which are the same as those of the test device described later. The displacement of the rotor was fixed to zero, and the limit of the coil current was set to $0.1 \mathrm{~A}$, which corresponds to about $0.17 \mathrm{~T}$ from Eq. (39).

Table 1 Parameters of six-pole AMB.

\begin{tabular}{lccl}
\hline Air gap & $g_{0}$ & $0.222 \times 10^{-3}$ & $\mathrm{~m}$ \\
Cross section & $S$ & $4 \times 10 \times 10^{-6}$ & $\mathrm{~m}^{2}$ \\
Coil turns & $N$ & 300 & \\
\hline
\end{tabular}

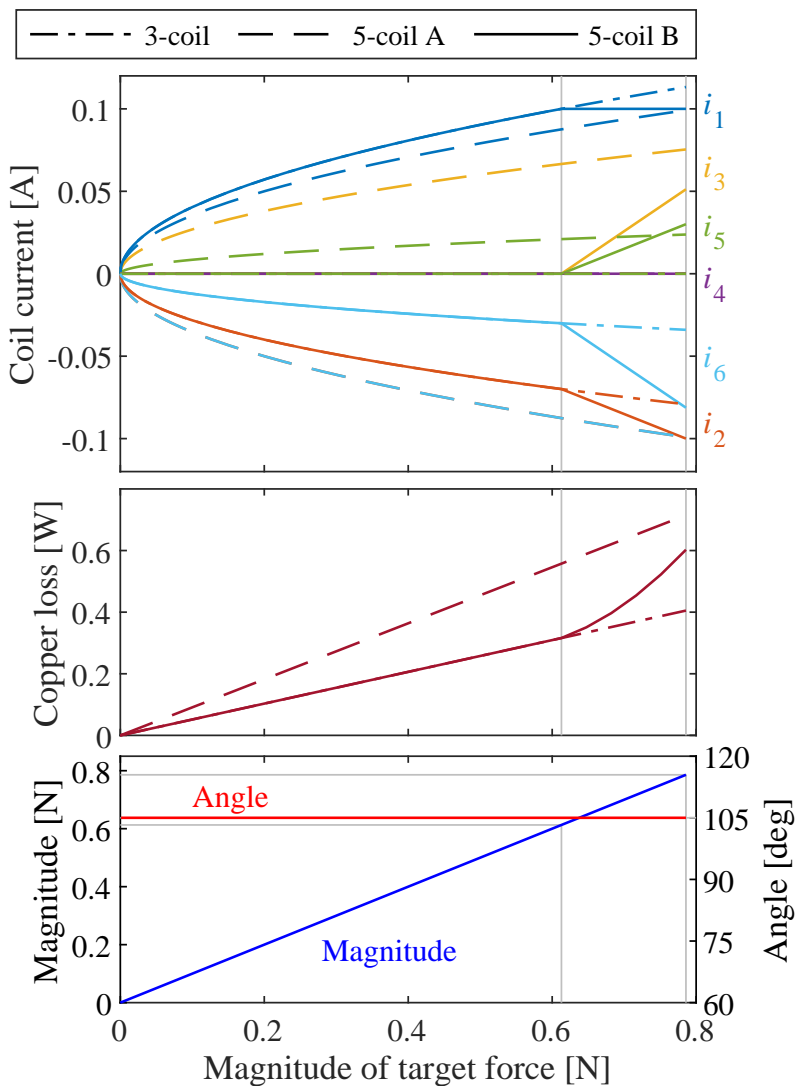

(a) Variations in magnitude with constant angle.
Table 2 Specifications of six-pole AMB.

\begin{tabular}{ll}
\hline Stator outer diameter & $\phi 38 \mathrm{~mm}$ \\
Stator inner diameter & $\phi 10.5 \mathrm{~mm}$ \\
Rotor outer diameter & $\phi 10.1 \mathrm{~mm}$ \\
Axial length & $10 \mathrm{~mm}$ \\
Pole width & $4 \mathrm{~mm}$ \\
Material & Silicon steel $(\mathrm{t} 0.5 \mathrm{~mm})$ \\
Coil & UEW, $\phi 0.1 \mathrm{~mm} \times 300$ turns, $20 \Omega$ \\
\hline
\end{tabular}
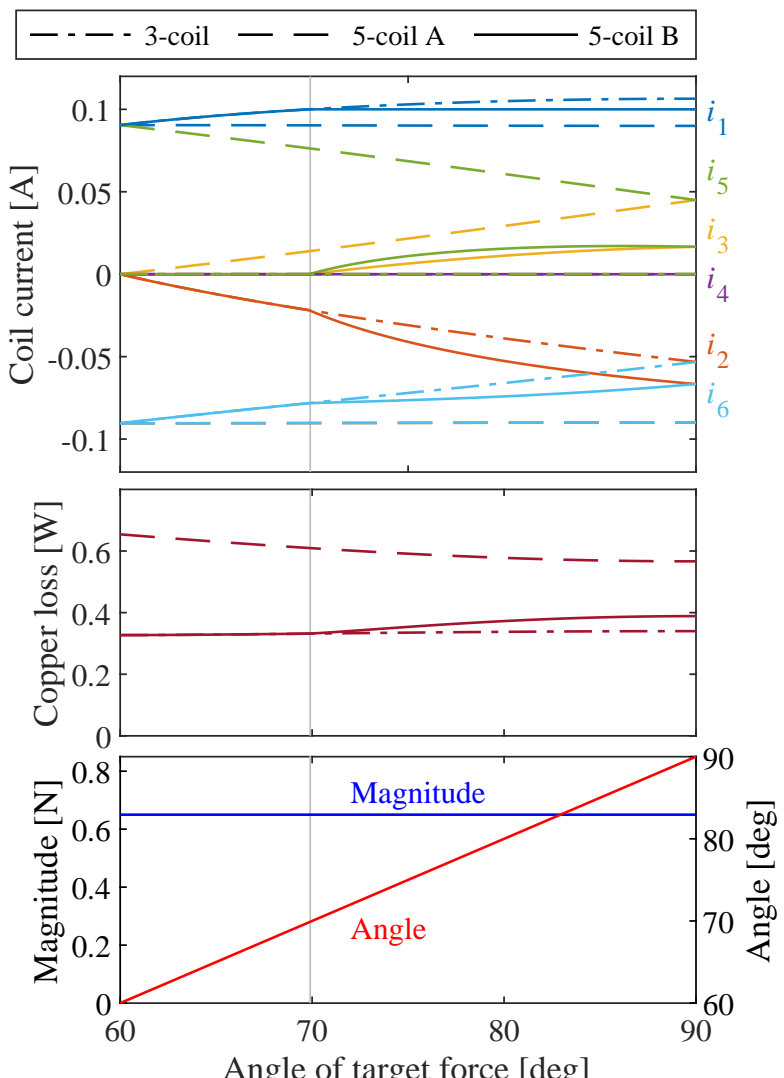

(b) Variations in angle with constant magnitude.

Fig. 2 Numerical verifications of the proposed methods. The upper graph shows the coil currents, the middle shows copper loss, and the lower shows the resultant force. Dash-dotted, dash and solid lines denotes the results of three-coil, five-coil A and five-coil B, respectively. These results confirmed that the target force can be generated by the proposed method. 


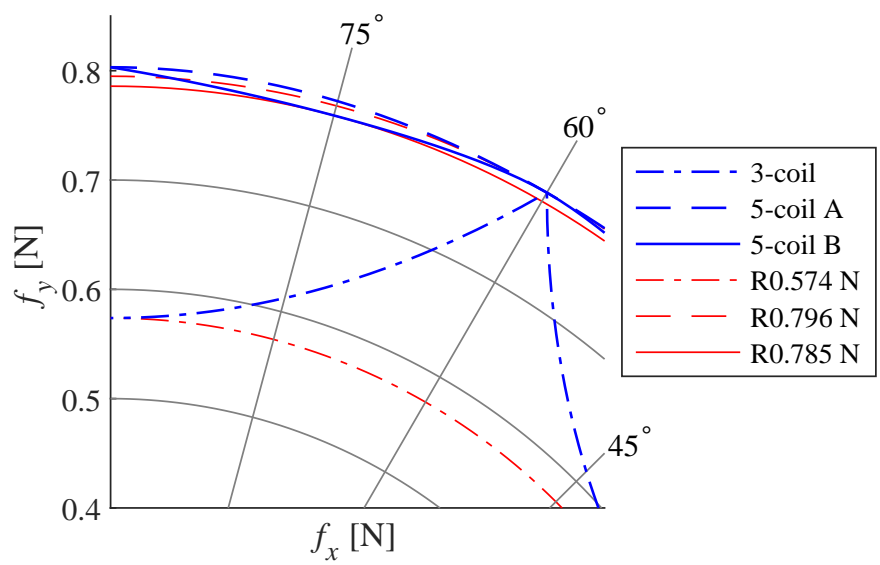

Fig. 3 Suspension force when the one of coils reaches maximum current $(0.1 \mathrm{~A})$. Blue lines indicate the maximum suspension force, and red lines indicate the inscribed circles of each mode. The grid lines indicate the amplitude and angle of the suspension force. When the target angle is $60^{\circ}$, the resultant force of all modes is the same. However, when the target angle is $90^{\circ}$, the resultant force of three-coil mode is much smaller than five-coil modes.

Figure 2 (a) shows the results when the magnitude of the target force was changed from 0 to maximum, while the target angle was set to $105^{\circ}$. When the target magnitude is less than $0.613 \mathrm{~N}$, the maximum current is less than 0.1 $\mathrm{A}$, and the suspension force can be obtained by the three-coil mode. When the target magnitude is greater than 0.613 $\mathrm{N}$, the maximum current in the three-coil mode exceeds the limit. However, the target force can be obtained by using five-coil modes. The currents in five-coil A mode jump from three-coil mode, while the currents in five-coil B mode change smoothly from three-coil mode to five-coil mode. Moreover, the copper loss in five-coil B mode is less than that in five-coil A mode.

Figure 2 (b) shows the results when the target angle was changed from $60^{\circ}$ to $90^{\circ}$ with a fixed magnitude of 0.65 N. From about $70^{\circ}$ to $90^{\circ}$, the three-coil mode exceeds the current limit. In contrast, the currents in five-coil modes are kept below the limit. Therefore, greater suspension force can be generated by switching from three-coil mode to five-coil mode.

Figure 3 shows the results of the maximum suspension force of three- and five-coil modes. The current limit was set to $0.1 \mathrm{~A}$. By using five-coil modes, a larger force can be generated. Red lines indicate the inscribed circles of each mode. The radii of five-coil A and B modes are 139\% and 137\% with respect to that of three-coil mode, respectively.

\section{Experimental verification}

\subsection{Test device and controller}

A test device is shown in Fig. 4. A rotor is set horizontally and six-pole AMBs are placed on each side of the rotor. A thrust magnetic bearing is not installed, and the passive stability of the AMBs is used to support the rotor along the axial

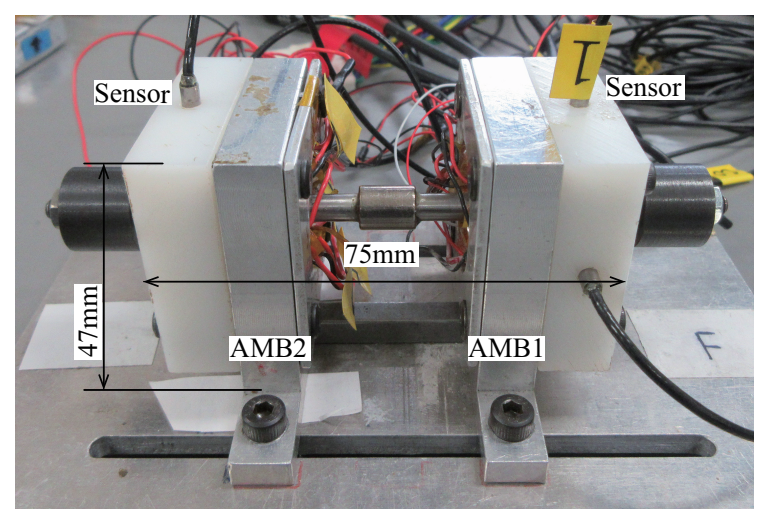

(a) Test device

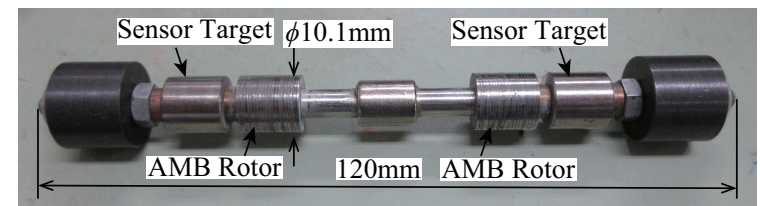

(b) Rotor

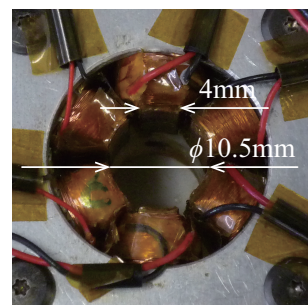

(c) Stator

Fig. 4 Photographs of test device. (a) is the whole device, (b) is the rotor and (c) is the stator. The total weight of the rotor is $69 \mathrm{~g}$. The specifications of the AMB are listed in Table 2. 


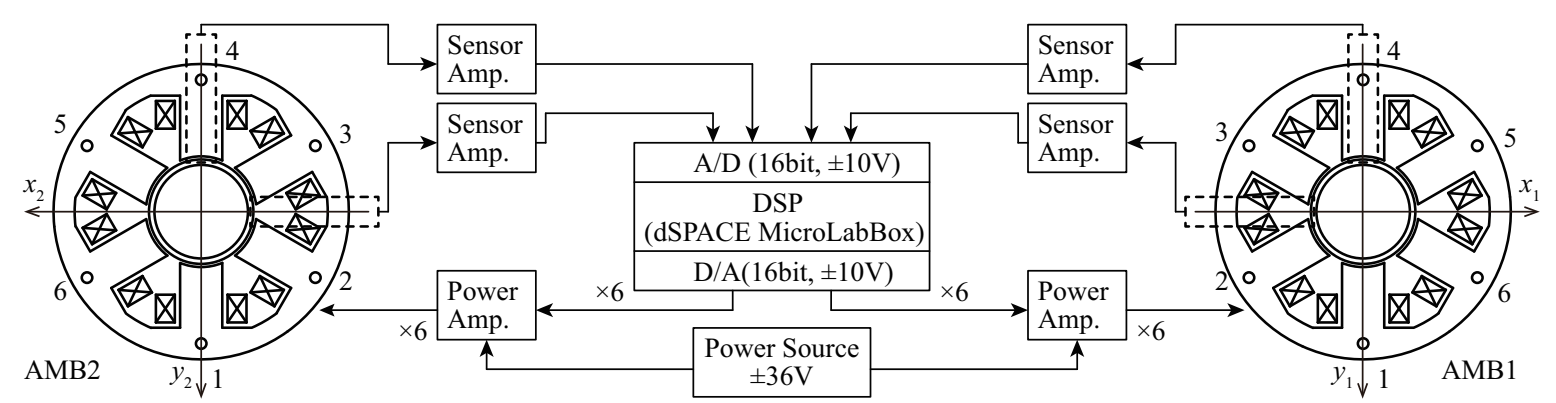

Fig. 5 Control system. Both AMBs are controlled by one DSP. MATLAB/Simulink is used for programming. The power amplifiers output the regulated currents due to a current feedback circuit.

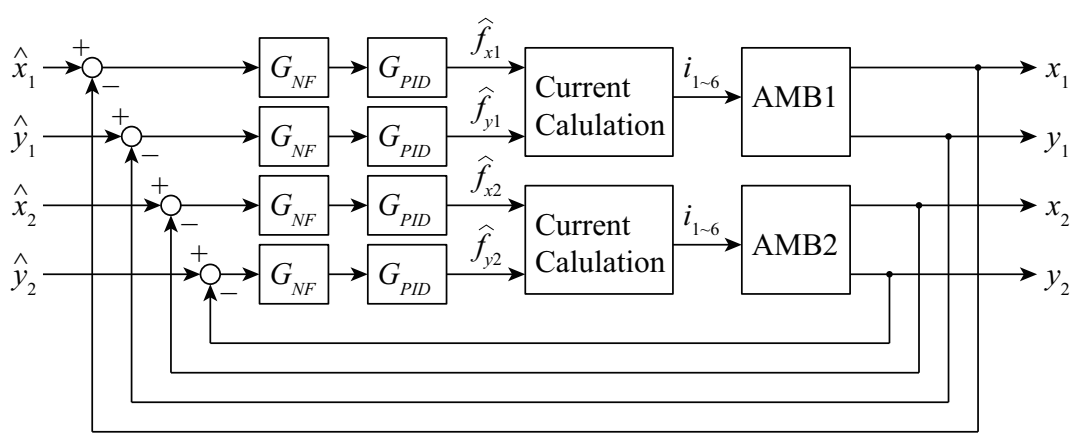

Fig. 6 Block diagram of controller. Each AMB is separately controlled. The gains of the PID controllers were experimentally determined.

Table 3 Parameters of controller.

\begin{tabular}{|c|c|c|c|c|c|c|c|}
\hline Proportional gain & $K_{P}$ & $20 \times 10^{3}$ & $\mathrm{~N} / \mathrm{m}$ & Cut-off frequency of notch filter & $\omega_{c}$ & $2 \pi \times 835$ & $\mathrm{rad} / \mathrm{s}$ \\
\hline Integral gain & $K_{I}$ & $10 \times 10^{3}$ & $\mathrm{~N} / \mathrm{ms}$ & Sampling time & $\tau$ & $0.1 \times 10^{-3}$ & $\mathrm{~s}$ \\
\hline Differential gain & $K_{D}$ & $0.05 \times 10^{3}$ & $\mathrm{Ns} / \mathrm{m}$ & Threshold for integrator & & 1 & $\mathrm{~N}$ \\
\hline
\end{tabular}

axis. Four displacement sensors are installed on the outside of the AMBs to detect the displacement of the rotor. Eddy current type displacement sensors (Omron: ZX-EDR5) are adopted.

A control system is shown in Fig. 5. A digital signal processor (DSP, dSPACE: MicroLabBox) is used for control. The DSP reads the displacement signals via A/D converters and calculates the coil currents of the AMBs. Then the DSP outputs the current commands via D/A converters to power amplifiers. Power Op Amp (TI, OPA548) is adopted for the power amplifier. The bandwidth of the amplifier was about $2.7 \mathrm{kHz}$.

Figure 6 shows the controller for the AMBs. The following proportional-integral-differential (PID) controllers are adopted for the position control.

$$
G_{P I D}=K_{P}+\frac{K_{I}}{s}+K_{D} \frac{s}{s / \omega_{b}+1}
$$

where $K_{P}, K_{I}$ and $K_{D}$ are proportional, integral and differential gains, respectively, and $\omega_{b}$ is break angular frequency of an approximate differentiator. Anti windup integrators are adopted. The integrator works when the output of $K_{P}$ is less than the threshold. To avoid spillover instability due to the flexible mode of the rotor, the following notch filter is inserted before the PID controllers.

$$
G_{N F}=\frac{s^{2}+\omega_{c}^{2}}{s^{2}+\sqrt{2} \omega_{c} s+\omega_{c}^{2}}
$$

where $\omega_{c}$ is cut-off angular frequency. These transfer functions are converted to discrete transfer functions by Tustin's method with sampling time $\tau$, and implemented with the DSP. The parameters of the controller are shown in Table 3. They are experimentally determined.

The coil currents of the six-pole AMBs are calculated by three-coil mode alone, five-coil mode alone, switching between three-coil and five-coil A modes or switching between three-coil and five-coil B modes. 


\subsection{Levitation experiments}

Figure 7 shows the results of levitation experiments when the limit of the coil current was gradually reduced from 0.15 A to 0 A because it was difficult to apply large load to the AMBs.

As shown in Figs. 7 (a) and (b), stable levitation could be realized with a lower current limit in five-coil A mode alone compared with three-coil mode alone. In contrast, copper loss with five-coil A mode alone was higher than that with three-coil mode alone when the target force was less than that of the three-coil mode alone maximum. Average target forces with three- and five-coil modes were measured as Table 4. There were errors between two modes. One of the possible reasons is misalignment and assembling error of the device. These results indicate that the generated forces varied with three- and five-coil modes with the same target forces.

The switching control strategy achieved both a lower current limit and less copper loss as shown in Figs. 7 (c) and (d). However, the repeatedly switching between three- and five-coil A modes and the vibration of the rotor appeared in Fig. 7 (c). Since the generated forces were varied with three- and five-coil A modes, impulsive force was applied to the rotor at the instance of switching, then the vibration was excited. The vibration affected the target forces, hence the repeated mode change was occurred.

In contrast, the repeatedly switching did not appear in Fig. 7 (d). In this case, the currents were changed smoothly from three-coil mode to five-coil B mode, moreover, the target forces gradually varied according to the current limit. Therefore, the impulsive forces were not applied, then the switching did not affect the displacements. The oscillations of the currents and copper loss with the five-coil B mode were larger than those with the three-coil mode. In the five-coil B mode, the control currents increase with decreasing the bias currents as shown in Eqs. (25) and (26), and the bias currents depend on the current limit as shown in Eq. (31). Therefore, the oscillation increased with decreasing the current limit, however, did not affect the displacements.

Figure 8 shows the results when a step signal was injected into the reference displacement $\hat{y}_{2}$. Under steady-state conditions, the three-coil mode was adopted at $-12 \mu \mathrm{m}$, while the five-coil mode was adopted at $12 \mu \mathrm{m}$. Like the previous results, the chattering and vibration appeared by switching between three-coil and five-coil A modes. In contrast, the chattering and vibration were reduced by switching between three-coil and five-coil B modes. The overshoot at $12 \mu \mathrm{m}$ was much larger than that at $-12 \mu \mathrm{m}$. One of possible reason is that the suspension force was different from the desired value due to the influence of the rotor displacement, which was not considered in the current calculation. This is a problem for future investigation.

The results of the levitation tests confirm that the switching strategy allows for both a larger suspension force and lower copper loss. Moreover, the results show that the switching between three-coil and five-coil B modes provides better control performance compared to the switching between three-coil and five-coil A modes.

Table 4 Target suspension forces of AMB2 with three- and five-coil A modes.

\begin{tabular}{c|cccc} 
& $\hat{f}_{x 2}[\mathrm{~N}]$ & $\hat{f}_{y 2}[\mathrm{~N}]$ & $\left|\hat{f}_{2}\right|[\mathrm{N}]$ & $\hat{\phi}_{2}\left[^{\circ}\right]$ \\
\hline Three-coil mode & -0.0125 & -0.597 & 0.597 & -91.2 \\
Five-coil A mode & 0.0265 & -0.529 & 0.530 & -87.1
\end{tabular}

\subsection{Start-up experiment}

Generally, magnetic attractive force becomes weak when the air gap becomes large. This requires larger currents for the start-up from the touch-down position compared to steady-state levitation. When the large current cannot be applied due to the current limit, the rotor cannot levitate directly from the touch-down position. To avoid this problem, we propose an alternative start-up method as follows.

Firstly, the currents that are calculated from the constant target force are supplied. If the target angle faces toward a narrower air gap pole, the rotor starts to move to that pole because it is possible to generate greater magnetic attractive force. Then the rotor will then be settled to different position. If the required currents become less than the current limit, then the rotor can levitate from that position.

Figure 9 shows the results of the start-up experiment. The coil currents were zero at first, then the constant currents with $\hat{f}=0.8[\mathrm{~N}]$ and $\hat{\phi}=-70^{\circ}$ were applied from $t=0$. The rotor then started to move, and settled in a different position, which is higher vertically. The rotor could levitate from this position, and the start-up was achieved. As shown in this experiment, it is possible to start the levitation control with a low current limit. 

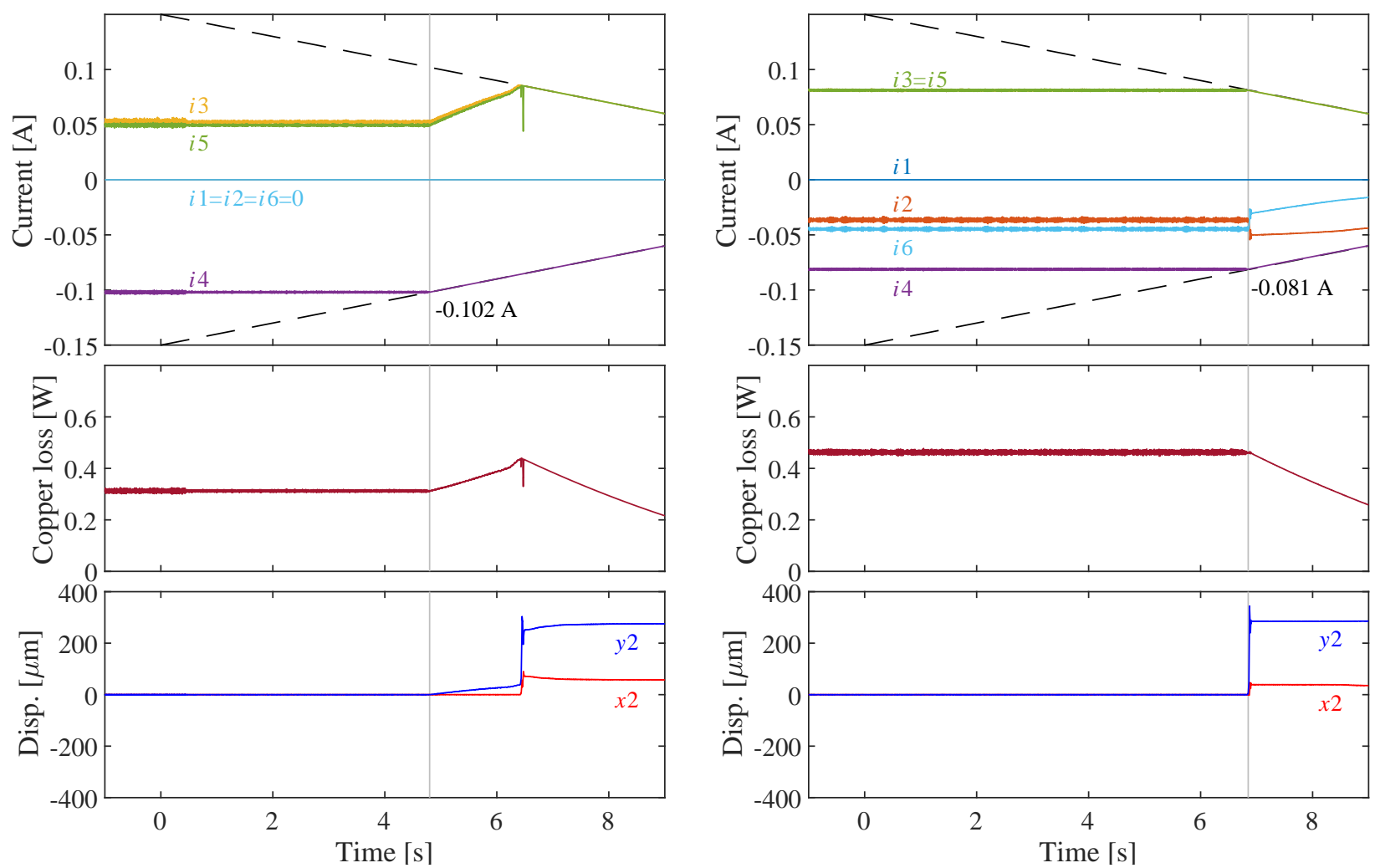

(a) three-coil mode alone.

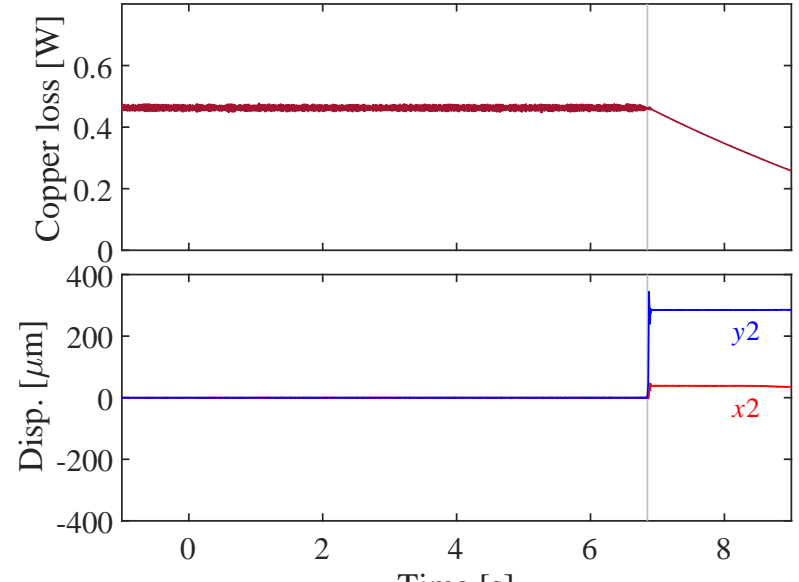

(b) five-coil A mode alone.
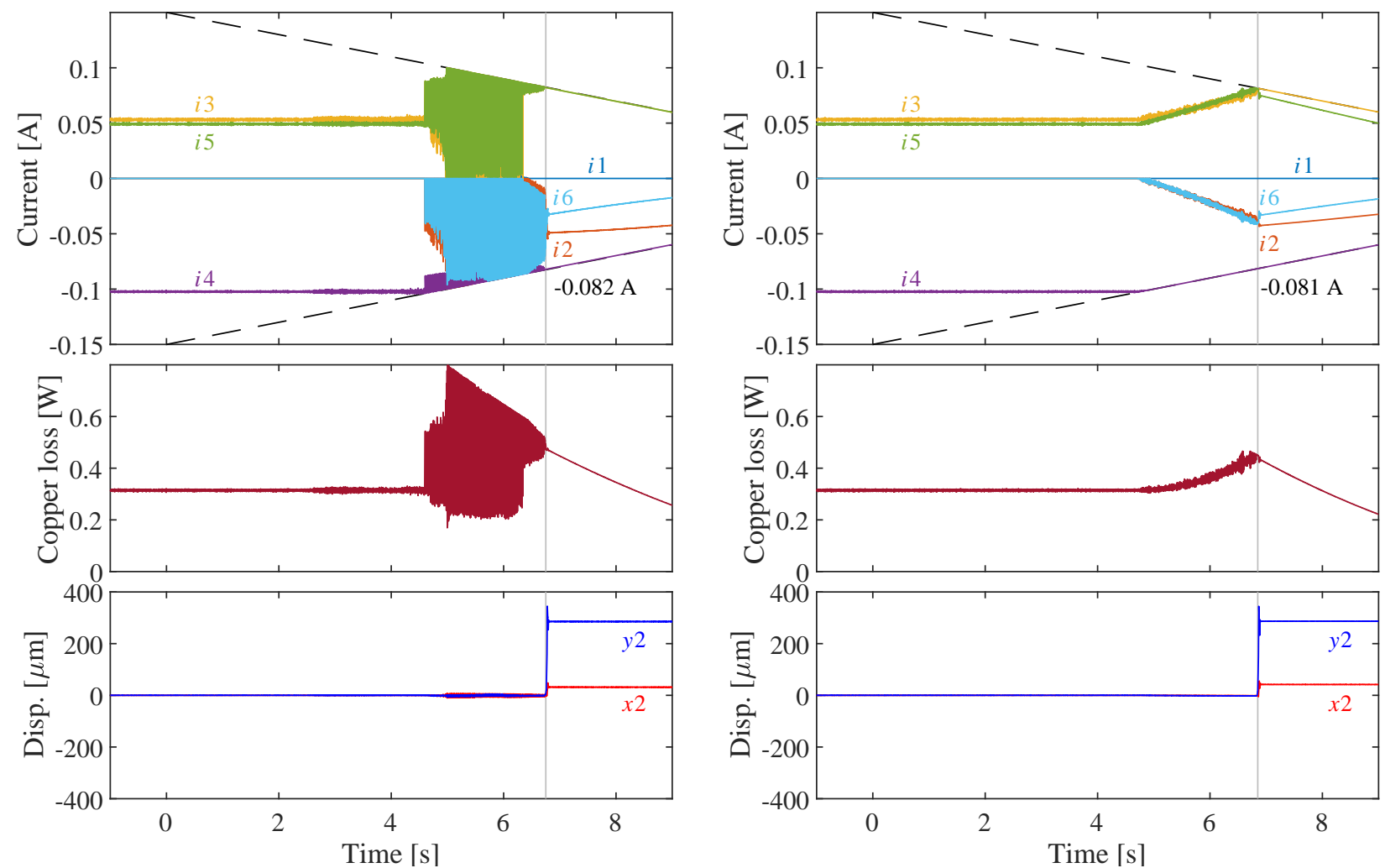

(c) Switching between three- and five-coil A modes.

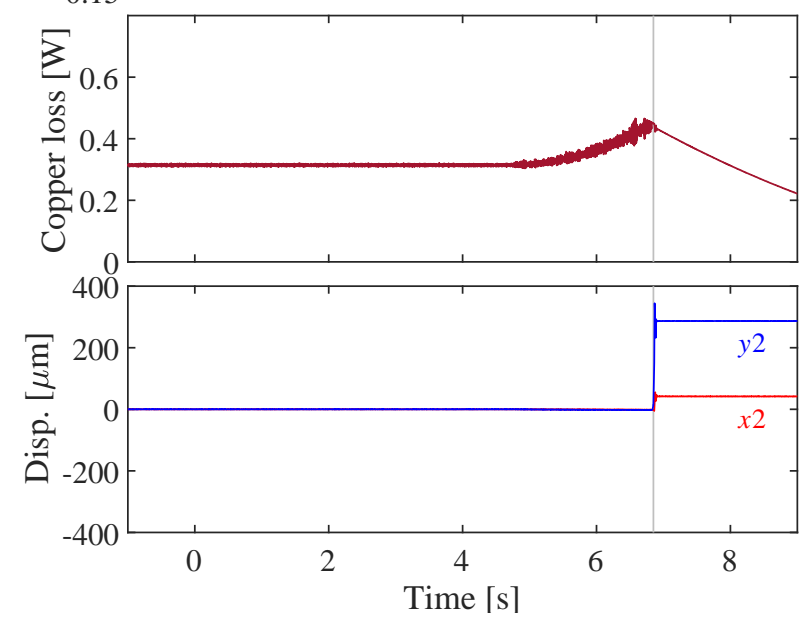

(d) Switching between three- and five-coil B modes.

Fig. 7 Currents and displacements of AMB2 when the current limit was gradually reduced from $0.15 \mathrm{~A}$ to $0 \mathrm{~A}$. The upper graph shows coil currents, the middle shows copper loss, and the lower shows the displacements of the rotor. Dashed lines indicate the current limit. Gray vertical lines show the time when the position control failed, and corresponding current limits are indicated in figures. 

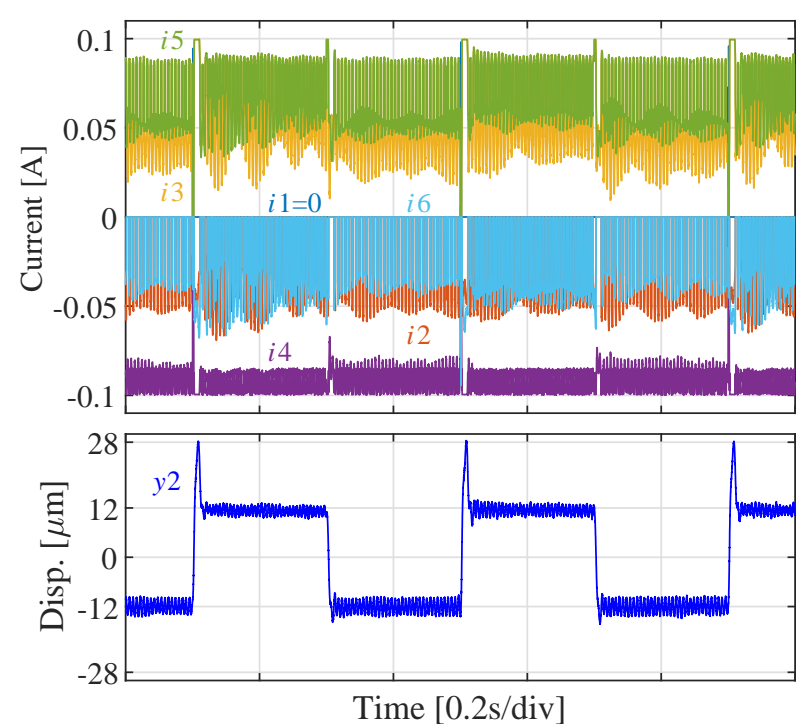

(a) Switching three- and five-coil A modes.
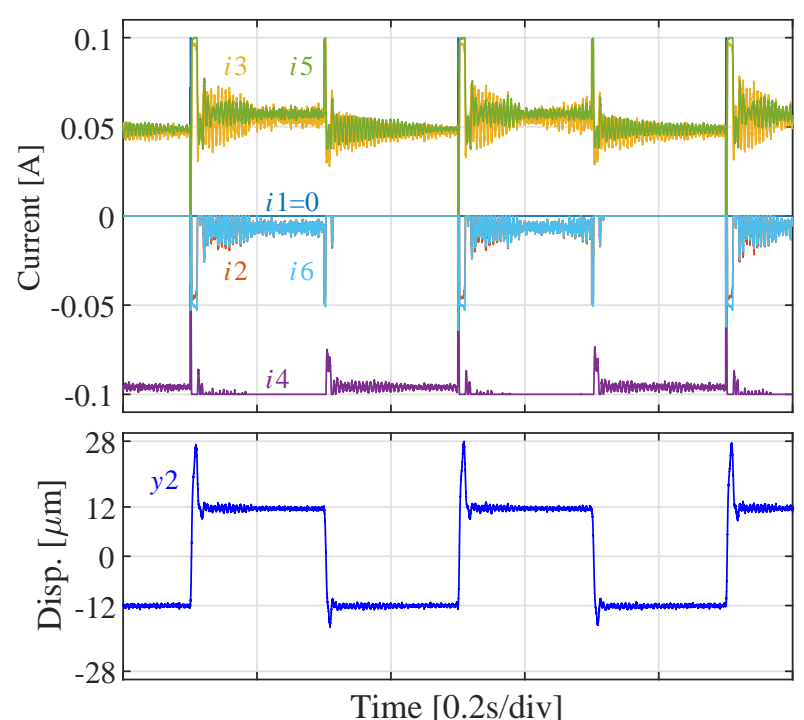

(b) Switching between three- and five-coil B modes.

Fig. 8 Step responses for reference displacement. The current limit was set to $0.1 \mathrm{~A}$. The chattering and vibration appeared in (a), while they were reduced in (b).

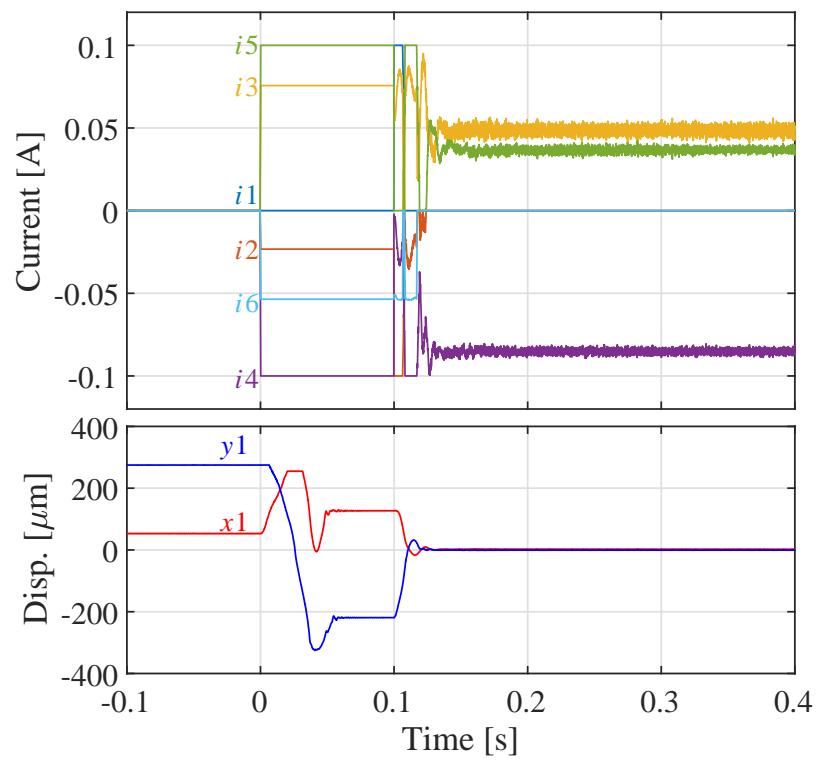

(a) AMB 1.

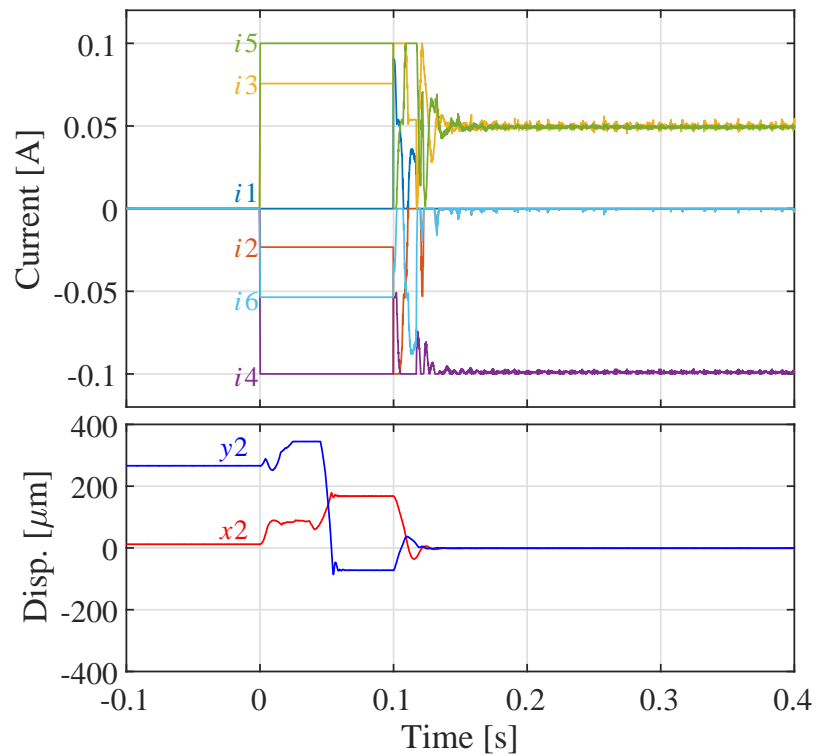

(b) AMB 2.

Fig. 9 Start-up control with current limit of $0.1 \mathrm{~A}$. Constant currents were applied at $t=0[\mathrm{~s}]$, and position control was started from $t=0.1$ [s]. Although the rotor did not levitate when the levitation control was started at the initial position, the rotor could levitate by using the proposed procedure.

\section{Conclusions}

In this paper, we introduced a novel current calculation method for six-pole AMBs, which switches three-coil and five-coil modes to realize both low power consumption and a large suspension force. The experimental results showed that the proposed method achieved stable levitation with a lower current limit compared to three-coil mode alone, and lower power consumption compared with five-coil A mode alone. Moreover, we proposed the start-up procedure with a low current limit, and experimentally confirmed the effectiveness of the proposed procedure.

\section{References}

Asama, J., Watanabe, D., Oiwa, T. and Chiba A., Development of a one-axis actively regulated bearingless motor with a repulsive type passive magnetic bearing, Proceedings of the 2014 International Power Electronics Conference (2014), 
pp. 988-993.

Chen, S. and Hsiao, Y., Smooth voltage controller and observer for a three-pole active magnetic bearing system, Mechanical Engineering Journal, Vol. 4, No. 5 (2017), Paper No.16-00718.

Chiba, A., Fukao, T., Ichikawa, O., Oshima, M., Takemoto, M. and Dorrell, D.G., Magnetic bearings and bearingless drives, Newnes (2005).

Chiba, M., Ueno, S. and Jiang, C., Performance comparison of 6 salient-pole active magnetic bearings by the difference in the control method, Journal of the Japan Society of Applied Electromagnetics and Mechanics, Vol. 25, No. 2 (2017), pp. 174-179. (in Japanese)

Kakihara, K., Koyanagi, H. and Okada, Y., Development of built-in PM type hybrid magnetic bearing, Transactions of the Japan Society of Mechanical Engineers, Series C, Vol. 71, No. 710 (2005), pp. 2968-2974. (in Japanese)

Kanebako, H. and Okada, Y., New design of hybrid type self-bearing motor for high-speed miniature spindle, Proceedings of The Eighth International Symposium on Magnetic Bearings (ISMB8) (2002), pp. 65-70.

Li, L., Shinshi, T., Kuroki, J., and Shimokohbe, A., A simple and miniaturized magnetic bearing for cost-sensitive applications, Proceedings of The Eighth International Symposium on Magnetic Bearings (ISMB8) (2002), pp. 561-56.

Mizuno, T. and Higuchi, T., Control of magnetic bearings using the observer for unbalance, IEEJ Transactions on Electrical and Electronic Engineering, Vol. 110-D, No.8 (1990), pp. 917-924.

Osa, M., Masuzawa, T., Saito, T. and Tatsumi, E., Magnetic levitation performance of miniaturized magnetically levitated motor with 5-DOF active control, Mechanical Engineering Journal, Vol. 4, No, 5 (2017), Paper No. $17-00007$.

Ueno, S., Arai, T. and Odagiri, K., Control of an active magnetic bearing with 6 concentrated wound poles using bias current, Journal of the Japan Society of Applied Electromagnetics and Mechanics, Vol. 12, No. 1 (2004), pp. 54-61. (in Japanese)

Ueno, S., Arai, T. and Odagiri, K., Minimum energy control of 6 salient-pole type active magnetic bearing, Journal of the Japan Society of Applied Electromagnetics and Mechanics, Vol. 15, No. 1 (2007), pp. 34-41. (in Japanese)

Ueno, S., Maximum bearing force control for 6 salient-pole active magnetic bearings, Transactions of the Japan Society of Mechanical Engineers, Series C, Vol. 79, No. 801 (2013), pp. 1475-1482. (in Japanese)

Yoshida, T., Ueno, S., Okada, Y., Arai, T. and Odagiri, K., Linearization control of 3 pole internal PM type hybrid magnetic bearings, Journal of the Japan Society of Applied Electromagnetics and Mechanics, Vol. 14, No. 4 (2006), pp. 406-412. (in Japanese) 